approach, and simple observation with high-dose intravenous antibiotics. ${ }^{5}$ In a critical situation such as this case, however, less invasive treatment might be advisable. Urgent cerebral angiography and an endovascular approach to mycotic aneurysms, especially for solitary and peripheral lesions, is an acceptable option for some patients with active endocarditis and severely damaged hearts.

\section{REFERENCES}

1. Bohmfalk GL, Story JL, Weissinger JP, Brown WE. Bacterial intracranial aneurysms. J Neurosurg 1978;48: 369-82.
2. Salgado AV. Central nervous system complications of infective endocarditis. Stroke 1991;22:1461-3.

3. Hart RG, Kagan-Hallet K, Joerns SE. Mechanisms of intracranial hemorrhage in infective endocarditis. Stroke 1987;18:1048-56.

4. Frizzell RT, Vitek JJ, Hill DL, Fisher WS 3rd. Treatment of a bacterial (mycotic) intracranial aneurysm using an endovascular approach. Neurosurgery 1993; 32:852-4.

5. Moskowitz MA, Rosenbaum AE, Tyler HA. Angiographically monitored resolution of cerebral mycotic aneurysms. Neurology 1974;24:1103-8.

\title{
COMBINED CORONARY ARTERY BYPASS GRAFTING AND EXCISION OF ADRENAL PHEOCHROMOCYTOMA
}

\author{
Peng W. Seah, MB, BCh, BAO, Robert Costa, MB, BS, and Hugh Wolfenden, FRACS, \\ Little Bay, New South Wales, Australia
}

The treatment of a patient with an adrenal medullary pheochromocytoma ${ }^{1}$ who also requires surgical revascularization for coronary artery disease presents a rare and interesting challenge. Previously documented experience with this combination of conditions is scarce, and to our knowledge this is the first reported case in which combined coronary artery bypass grafting (CABG) and excision of an adrenal pheochromocytoma was performed.

A 69-year-old woman had a history of an acute anterior myocardial infarction 3 months previously. Coronary angiography revealed severe triple-vessel disease, with an ejection fraction of $49 \%$ and association with anteroapical and inferior hypokinesia. She had New York Heart Association functional class IV angina and was receiving maximal antianginal therapy. Her medical background included a 3-year history of hypertension being treated with metoprolol at $100 \mathrm{mg}$ twice daily and lisinopril at 2.5 mg daily.

While in the hospital with unstable angina and awaiting surgical revascularization, the patient was examined for right upper quadrant abdominal pain and deteriorating liver function. Abdominal ultrasonography and computed tomographic scan (Fig. 1) revealed a well-defined mass measuring approximately $5 \mathrm{~cm}$ in diameter below the right lobe of the liver and arising from the right adrenal gland. Urinary catecholamine levels were elevated, and scintigraphy with metaiodobenzylguanidine labeled with iodine 131 showed this to be a functioning mass consistent with a

From the Department of Cardiothoracic Surgery, The Prince Henry Hospital, Little Bay, New South Wales, Australia.

J Thorac Cardiovasc Surg 1995;110:559-60

Copyright 1995 by Mosby-Year Book, Inc.

$0022-5223 / 95 \$ 3.00+0 \quad \mathbf{1 2 / 5 4 / 6 2 6 1 8}$ pheochromocytoma. An $\alpha$-adrenoceptor blockade was started with oral phenoxybenzamine and the dosage was stabilized at $20 \mathrm{mg}$ three times daily after 3 weeks; this dosage was limited by symptomatic postural hypotension and tachycardia. Oral $\beta$-adrenoceptor blockade was continued with metoprolol.

A combined surgical procedure was performed in cooperation with the urologists. In an effort to reduce perioperative bleeding, a low-dose regimen of aprotinin was given at a dosage of $500,000 \mathrm{U}$ before and during bypass, with a further $200,000 \mathrm{U}$ given after the bypass. The patient underwent cardiopulmonary bypass (CPB). With mild systemic hypothermia and intermittent cold blood cardioplegia, the left internal mammary artery was anastomosed to the left anterior descending artery and saphenous vein grafts were anastomosed to the marginal circumflex and distal right coronary arteries. CPB was then withdrawn with surprising ease. After decannulation, the cannulating tourniquets were snared without being tied to facilitate rapid reintroduction of CPB in case this should be needed to control extremes of blood pressure during removal of the pheochromocytoma. The median sternotomy was extended as a midline laparotomy to just above the umbilicus, splitting the fibrous central part of the diaphragm to just above the inferior vena cava. The left lobe of the liver and the duodenum were mobilized, the perinephric fascia were incised, and the pheochromocytoma was exposed. The drainage veins were clipped and divided, and the tumor was removed. The patient remained in hemodynamically stable condition throughout, with no major swings in blood pressure. The sternotomy and laparotomy wounds were routinely closed, and the patient returned to the recovery room in a stable and satisfactory condition. Throughout the postoperative course, the patient's blood pressure remained remarkably 


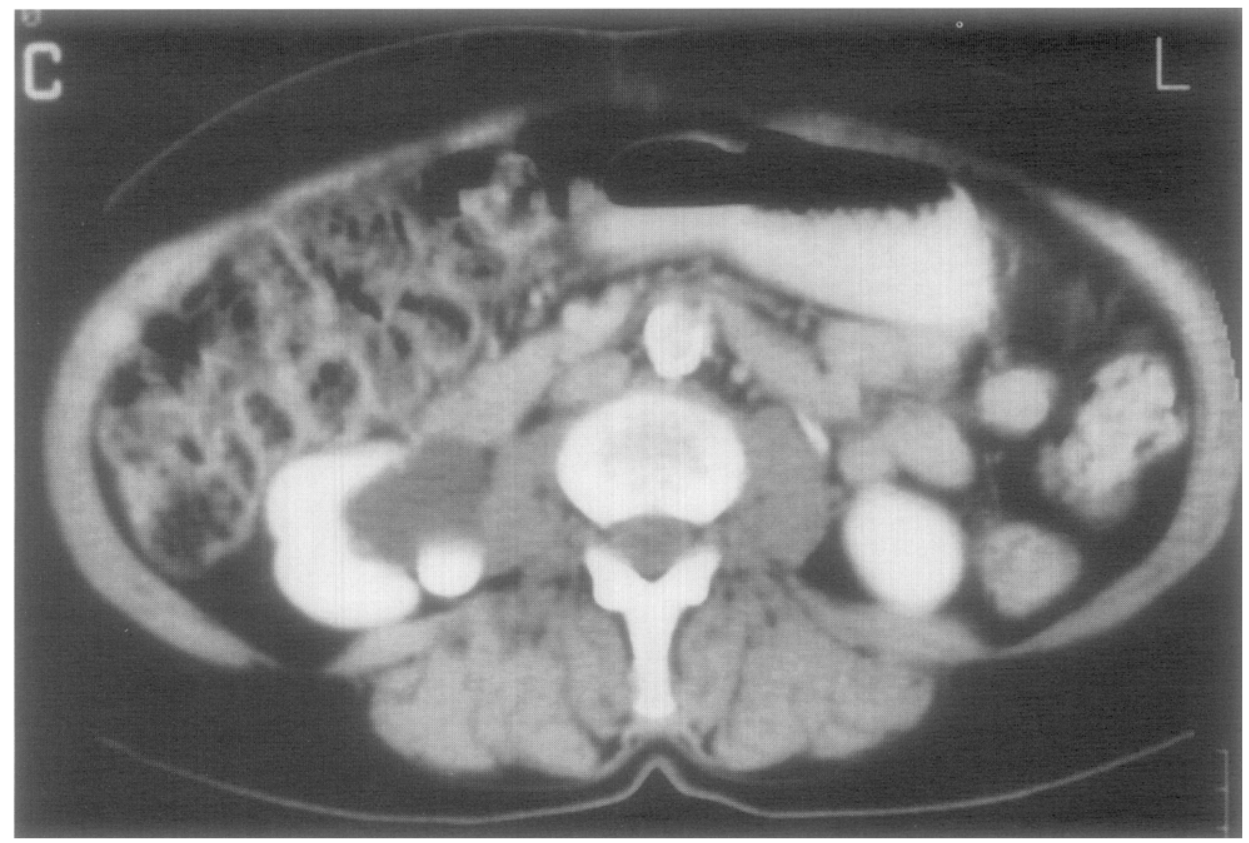

Fig. 1. Well-defined $5 \mathrm{~cm}$ right adrenal mass.

stable; she required only a minimal dose of sodium nitroprusside to control it. Her subsequent recovery was uneventful, and she was discharged from hospital on postoperative day 10 .

Vacheron and colleagues ${ }^{2}$ in 1984 proposed removing a pheochromocytoma first by ablation and only then to consider coronary angiography with a view to CABG. An extensive search of the medical literature failed to find any other instance in which CABG had been performed in conjunction with the excision of a pheochromocytoma. We believe this to be a viable management option, as demonstrated by the good result obtained for this patient. The perils of cardiovascular crises involving extreme paroxysmal swings of blood pressure, malignant tachyarrhythmias, pulmonary edema, and shock associated with CABG in the presence of a previously undiagnosed pheochromocytoma have all been well documented ${ }^{3,4}$ and are associated with a high mortality rate. Conversely, performing a laparotomy to remove a pheochromocytoma in the presence of unstable angina pectoris risks an acute perioperative myocardial infarct and perhaps even death. Either way, the perioperative course would probably be stormy, with a significant risk of death. For these reasons, we elected to proceed with a combined surgical procedure.

The tumor was shown to be both actively secreting (elevated urinary catecholamines) and metabolically active (results of scintigraphic scanning with metaiodobenzylguanidine labeled with iodine 131). The lack of hypertensive crises and other complications commonly associated with the removal of such a tumor was, we believe, caused by the effective combined $\alpha$ - and $\beta$-adrenoceptor blockade. The availability of $\mathrm{CPB}$ offered a further level of safety in case hemodynamic instability had occurred during the laparotomy. If necessary, the option of resection under profound hypothermic arrest was also available. This would have stopped all venous drainage from the tumor if it became difficult to control the wide swings of blood pressure commonly seen to result from mechanical manipulation. The combined procedure also offered the patient the advantage of a shorter overall length of hospital stay. Our patient's recovery was uneventful; she was back onto a normal dietary regimen and was fully ambulatory by postoperative day 6 .

To our knowledge, this case is the first documented instance of a combined operation for coronary artery disease and adrenal pheochromocytoma. It demonstrates the feasibility of a combined procedure, and we believe that this combination should be considered as a management option when dealing with a patient who has an adrenal pheochromocytoma and also requires coronary artery revascularization.

\section{REFERENCES}

1. Gough I, Thompson N. Phaeochromocytoma. Aust NZ J Surg 1988;58:365-8.

2. Vacheron A, Heulin A, Baubion N, et al. Phaeochromocytoma and aortocoronary bypass: double surgical procedure. Ann Med Interne 1984;135:305-7.

3. Dunn EJ, Wolff RK, Wright CB, et al. Presentation of undiagnosed phacochromocytoma during coronary artery bypass surgery. J Cardiovasc Surg 1989;30:284-7.

4. Brown P, Caplan RA. Recognition of an unsuspected phaeochromocytoma during elective coronary artery bypass surgery. Can Anaesth Soc J 1986;33:785-9. 